\title{
Performance Measure and Analysis of MPLS and conventional IP network through VoIP: Effect in Video and Audio transmission
}

Jawad Oubaha, Abderrahmane Ez-zahout

SIME Laboratory, ENSIAS University Mohammed V Rabat

Morocco

jawadoubaha@yahoo.fr

abderrahmane.ezzahout@um5s.net.ma

\begin{abstract}
For the mapping the network interconnection of the different technology MPLS backbone and the wireless network WiFi, we have only one choice to ensure the Quality of service the end-to-end. It is the service mapping considering as the future solution to ensuring the performance traffic engineering between Label Switch Path "LSP" of MPLS and access Category " $A C$ " of IEEE802.11e wireless. This paper will propose the mapping between these technologies with Fuzzy logic to optimize the quality of service in MPLS networks. MPLS and 802.11e is very useful approach for today's things, the internet of Big Data to keep a level of the Quality of service the end-to-end. It studies about different approaches to map 802.11e Access Categories (AC) to Label Switched Path (LSP) and their contributions. It then introduces the concept of encapsulated LSPS with Fuzzy to achieve future QoS, which requires an in-depth study to examine its practicability.
\end{abstract}

Keywords: MPLS, Fuzzy, 802.11e, Mapping, Audio, Video, Performance and Optimization

Received: 30 November 2017, Revised 11 January 2018, Accepted 29 January 2018

(C) 2018 DLINE. All Rights Reserved

\section{Introduction}

The varieties applications in the Internet Protocol are increasingly integrated in the business, job and also in multimedia traffic in the transmission of real time over the IP heterogeneous network [1][2].

This situation requires the classification of services by the resource sharing and assigning of priority to have the end-to-end quality of service. Best effort is an IP network service in which the network does not provide any guarantees data packet which is received where a user is given a guaranteed quality of service level or a priority. IP network is trying to improve its transmission and found solutions to adapt this growing demand by providing different services like VoIP (Voice over IP) and Video [13] [14], Differentiated Service (DiffServ) and MPLS (Multi Protocol Label Switching), and also it provides the new standard that improves the quality of service as IEEE 802.11e wireless network. In the first section $802.11 \mathrm{e}$ is explained. In order to manage the transmission packet and create the backup paths, MPLS develops the differentiated services and the speed treatment of layer 2 switching in IP network. MPLS is a promising solution to take over the next generation IP networks, where the section 2.2 explains the MPLS protocol. We will exploit many notions of treatment in common, MPLS can be combined with IEEE 802.11e to provide the quality of service along with the network with traffic engineering. Section 3 discusses the IEEE combination 802.11e MPLS networks. Section 4, discussion describes the concept of LSP "label switch path" for future QoS needs. The end section 5 is experimentation model L-LSP_E-LSP and Section 6 concludes the paper.

\begin{tabular}{llllll}
\hline 56 & Journal of Networking Technology & Volume & 9 & Number 2 & June \\
\hline
\end{tabular}




\section{Mapping Context}

\subsection{QoS Support Mechanism of IEEE 802.11e}

To differentiate service according to its priority there are wireless priority model currently under discussion. IEEE Group currently defines some enhancements for 802.11 in the MAC level, called 802.11e which proposes EDCF and EPCF [3]. Stations which operate under 802.11e are names enhanced stations and an developed access point which may also work as the centralized controller for all other stations within the same BSSS is named the Hybrid Coordinator (HC). BSS contains an 802.11e, HC and stations such as an $802.11 \mathrm{e}$ AP contains the HC. In the next paragraph, we discuss the functionality $802.11 \mathrm{e}$ enhanced. The EDCF must be used in the CP only while the EPCF may used in both phases, which creates this new coordination function hybrid.

\section{Enhanced Distributed Coordination Function}

EDCF is a new version of DCF also uses the CSMA/CA. The contention access method to the channel is names EDCA (Enhance Distributed Channel Access).

\section{Access Categories (AC) parameters in EDCA}

- Four AC with different parameters exist with EDCA, they are designated by the flows that use them: AC_VO (voice), AC_VI (video) AC_BE (Best Effort) and AC_BK (Background).

- Replaces DIFS (Distributed Inter Frames Spacing) by AIFS [AC] (Arbitration IFS), with : AIFS [AC] = SIFS + AIFSN [AC] $\mathrm{x}$ SlotTime, where: AIFSN[AC] $>2$.

With AIFS[AC] > DIFS and AIFSN[AC] has the lowest value for the highest priority )Audio = video, data). (Figure 2).

- $\mathrm{CWmin}[\mathrm{AC}]$ and $\mathrm{CWmax}[\mathrm{AC}]$ vary with the $\mathrm{AC}$ according to application $\{$ Audio $=$ Video $<$ Data $)$.

- Persistence Factor PF, in the first, differs then set the default value2: With CWnew $=(C W o l d+1) \times$ PF-1[3].

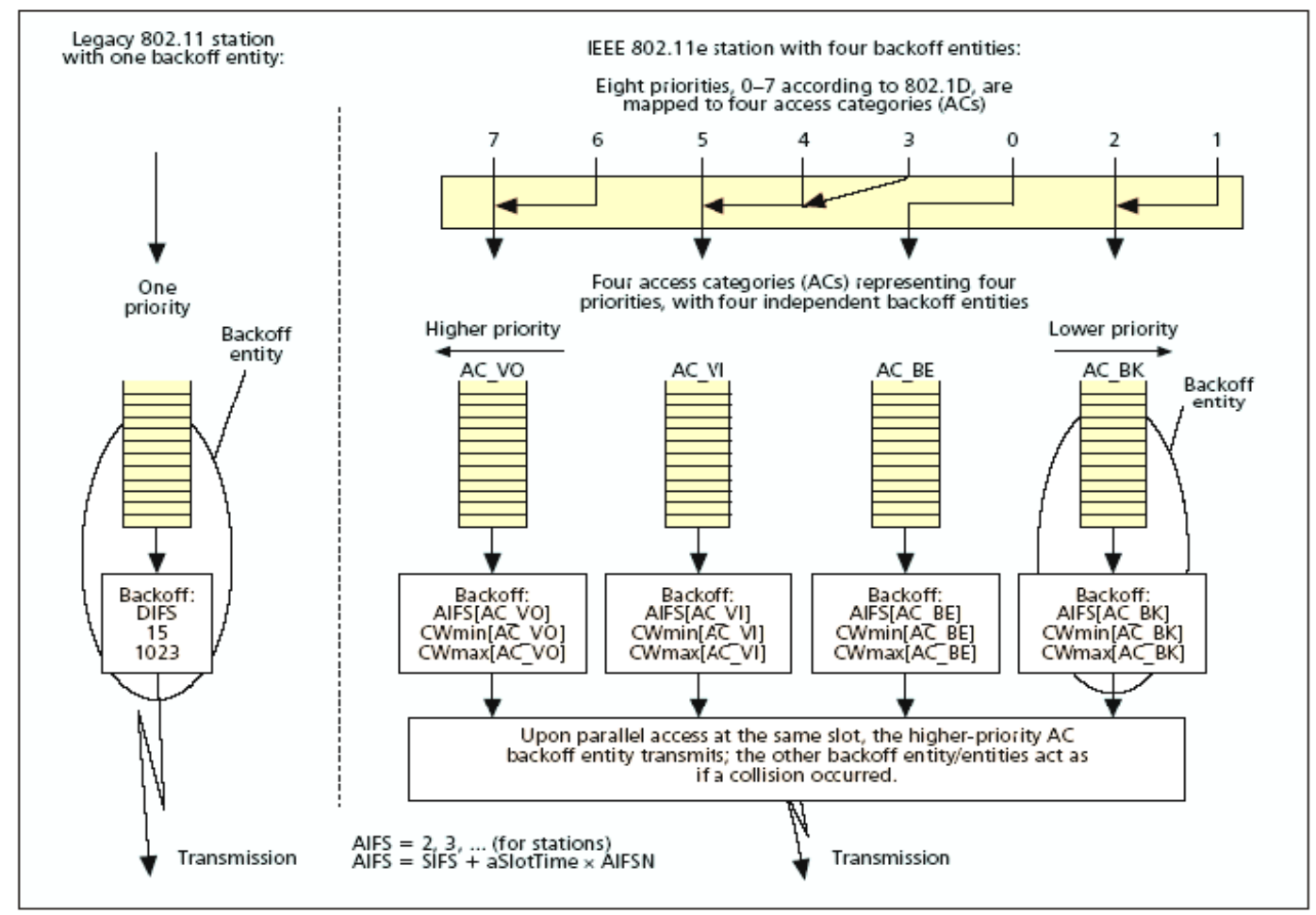

Figure 1. 802.11 And 802.11e stations with four ACs by station 


\begin{tabular}{|l|l|l|l|}
\hline Label (20 bits) & $\begin{array}{l}\text { Exp } \\
(3 \mathrm{bits})\end{array}$ & $\begin{array}{l}\text { S } \\
(1 \mathrm{bit})\end{array}$ & TTL (8 bits) \\
\hline
\end{tabular}

Figure 2. MPLS header

\subsection{Fuzzy Logic Wireless Multipath Routing (FLWMR)}

In FLWMR, the hop count is represent the QoS metric for route selection. The main functionality when a source host wants to send a message to a destination, it first calls upon the local fuzzy logic controller to define whether to send the message or drop it. If the decision is to send the traffic, FLWMR floods the network with route request packets (RREQ) to explore multiple paths to the destination [9]. When the destination receives the first request packet, it records path and returns a route reply (RREP) packet to the source via that path.

The destination then waits for a random time to receive other RREQ messages in order to discover additional routes that are disjoint from the first one. The multipath received, by the source of packet are added to path pool for the use by the fuzzy router. When a node detects a link break, it is not necessary to do route discovery again since FLWMR stores multiple routes to the destination. The fuzzy controller then decides to use the stored paths for the offered traffic according to the traffic importance and network status.

Fuzzy logic wireless load aware multipath routing (FLWLAMR) also chooses the route with criteria the least delay as the primary route for delivering packets between the source and the destination node, the second route is the path which is the maximally disjoined path with the primary one and has the shortest distance end to end [9]. The fuzzy routing algorithm reides at the source node monitors the congestion status of active routes, and feeds the network status to the fuzzy logic controller in order to make the best routing decision. The network status is measured as the number of packets buffered eat each node's interface. When the RREQ packet reaches the destination, it calculates the network status by measuring the number of packets buffered in each intermediate note in the network and sends back to the source with each RREP. Both FLWMR and FLWAMR dynamically allocate the network bandwidth depending on the priority of the packets and the status of the network, in the service agreement. This functionality encourages us to integrate the Logic Fuzzy in MPLS network.

\subsection{Multi Protocol Label Switching}

MPLS is developed to reduce the cost of routing network layer while giving it better performance, greater scaling with flexibility in restoration services network layer, by using labels. In a MPLS network, incoming packets are assigned a "label" according to their forwarding equivalence class (FEC) [7]. Packets are forwarded along a routers based solely on the contents of the label, eliminating the need to look for its IP address. These created paths. Label Switch Paths (LSP) can guarantee a minimum level of performance to create IP tunnels for network-based virtual private networks, VPN. This technology can more easily integrate technologies such as QoS, VPNs or VoIP, makes it a flagship technologies of internet of things already appreciated by the majority of Internet service providers as well as by customers. This evolution has encouraged the different networks type which at the same time increased the complexity of overall management. In terms of improvements, MPLS allows better result of routing, switching and transferring packages through networks of new generation [9].

The architecture uses a MPLS mechanisms switching Layers 2 of the OSI model with the routing layers 3 of the OSI model.

MPLS bases on two distinct components to reach its decisions: the control plan and data plane.

For ensure the transmission of packets, MPLS is based on the following parameters [7][8]:

- Source and/or destination IP address.

-IPv4 Differentiated Service (DS) code point.

-IPv6 Flow label.

- Source and/or destination port numbers.

-IP protocol ID (PID).

$58 \quad$ Journal of Networking Technology Volume 9 Number 2 June 2018




\section{Mapping between MPLS \& 802.11e}

IEEE 802.11e and MPLS help to solve the IP quality problem. IEEE 802.11e uses the IP type of service field to classify traffic into different classes at the interconnection node to provide QoS. MPLS also classifies traffic into different classes for FECs to improve QoS. To the mapping between MPLS network and 802.11e, the ToS of a packet determines the route of the packet. MPLS IEEE 802.11e network combines these to have the best match traffic engineering and QoS [11], [12].

The quality of service is constituting a real reason to achieve our approach:

- Classification of traffic at edge routers.

- Transit routers treat packets according to the labels.

- Complexity is pushed to edge routers.

- Labeling of packets after classifying.

- Aggregation support.

- Labels are short and of fixed length.

When a IEEE 802.11e packet arrives into a MPLS network, the incoming traffic is mapped to appropriate LSP after the ingress LSR examining of a TOS field of IP diagram to check the 802.11 e priority information.

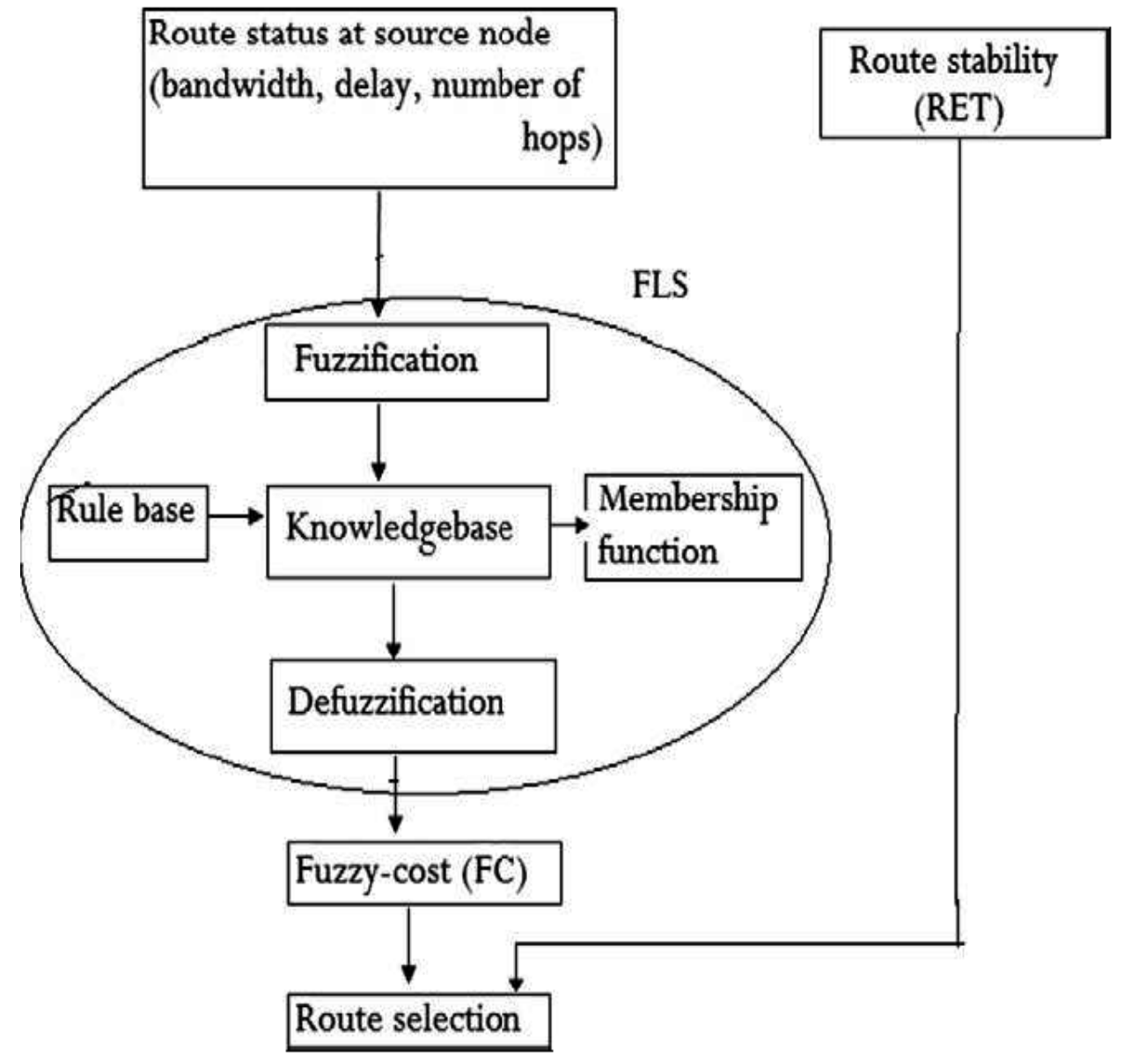

Figure 3. Selection of multi objective optimal route 


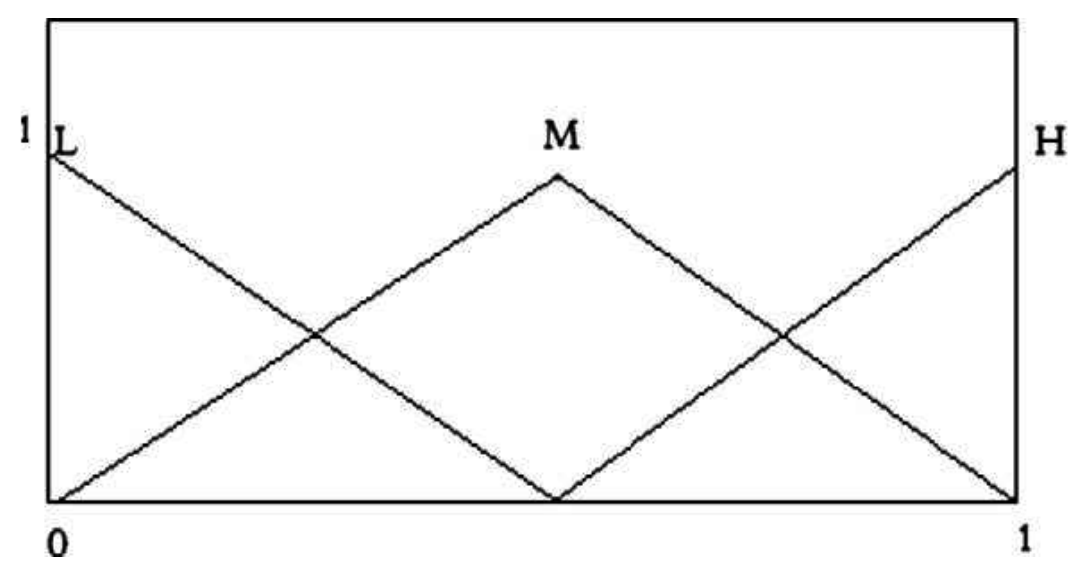

Figure 4. Fuzzy memberships function for bandwidth, delay and hop count

Figure 3 shows the fuzzy logic system (FLS) with the three major processes involved in: They are fuzzy inference, fuzzification and defuzzification [12]. The Fuzzy LS inputs are: (1) the number of intermediate hops, (ii) bandwidth and (iii) delay.

(a) Fuzzification of Inputs and Outputs: The three input variables to be fuzzified are bandwidth end-to-end delay and the number of intermediate nodes. On the existing knowledge of MANET, the terms "High", "Medium", "Low", are used to describe bandwidth, delay and the number of hops. For the output variable cost the terms "High", "Very High", "Low" and "Very Low" are used. Triangular membership functions as shown in Figure 4 is used for representing these variables.

(b) Knowledge Base Rule Structure: The fuzzy rules have IF-THEN structure. The inputs are then combined using the AND operator. This is an example of the input output mapping describing rules.

If (Hop Count is "Short") AND (Bandwidth is "High" AND ( End-to-End Delay is "Low") then the cost is "Very Low".

The interpretation is that minimum number of intermediate hops, maximum bandwidth and minimum delay are favorable inputs and thus it gives very low cost. The total number of possible fuzzy inference rules is $3 * 3 * 3=27$, because each input variable has three linguistic states.

\section{Analysis}

Though MPLS label path is the answer for MPLS IEEE 802.11e with many types of behaviour defined, the other side ToS field is very useful in a network with limited number of traffic classifications (until 8 classes). Hence in this approach the MPLS network with Fuzzy can provide a quality of service from the end -to-end and enhanced service.

In future, QoS is going to be must demanding enhanced methods to cope the Big Data. Internet variety requires high granularity of QoS which demand more granularity of ACs in 802.11e. Internet service providers have many customers who need a level of connections. The different labels associated can be used for other customer when one customer is not using the network. Hence the total number of lables used will not exceed the scope and will still support more numbers of traffic classifications. Traffic engineering capabilities and QoS are going to be the technology increasingly used, provided by MPLS. Currently if MPLS technology is expanded to provide such high granular 802.11e it is the use of encapsulated LSPs; ToS field IP of LSP decide the QoS and the other field deciding the path [13]. 802.11e can be extended to provide high granularity of QoS using MPLS.

Labels denoted could be static or dynamic with Fuzzy learning. The static assignment means the fixed association of lable and service will reduce the memory load processing time of LSRs. By using the E-LSP, LSR know what how to treat the packet. LabelService association will not require LSRs to swap signaling messages with their neighbors [14].

Mainly the Internet Service Provider ISP must define the QoS-MPLS policy for the label-Service association. The signaling protocols forward this information to all other LSR using. 
In the case of dynamic assignment labels, labels specify a different classes of service which should also be exchanged through signaling protocols. For this approach each MPLS router is composed of two separate databases: one for path decisions (control panel) and the other for traffic classes (data plan) and the other for traffic classes (data plan). Use of L-LSPs and E-LSPS together eliminate the need of establishing different LSPs for different classes. Thus the need of elimination to maintain large number of labels as label as same labels can be used for forwarding decision as well as denoting different service. The L-LSP will determine the path and the E-LSP will determine the service. This approach further study to test its feasibility.

\section{Simulation}

In the first, we will present the experimentation of the interconnection that unites two technologies: IEEE 802.11e and MPLS. 802.11e that present here is the transmission system of data assuring the link between peripherals by the access point and MPLS represents the operator backbone that permits to simplify the administration by adding new interesting functionalities for the management of a needs transmission. We will discuss in a second time to give the result of the comparison between the two methods of creation of LSP: E-LSP and L-LSP.

The constitutation model of the MPLS-WiFi experimentation is:

- Two PC Windows that are connected via access points to the MPLS backbone.

- Two access points WiFi Linksys WAP54G 802.11g (54Mbps) of cisco.

- Three machines: 2LER and a LSR that present the MPLS backbone.

The figure 5 shows machine name, the IP addresses and used interfaces.

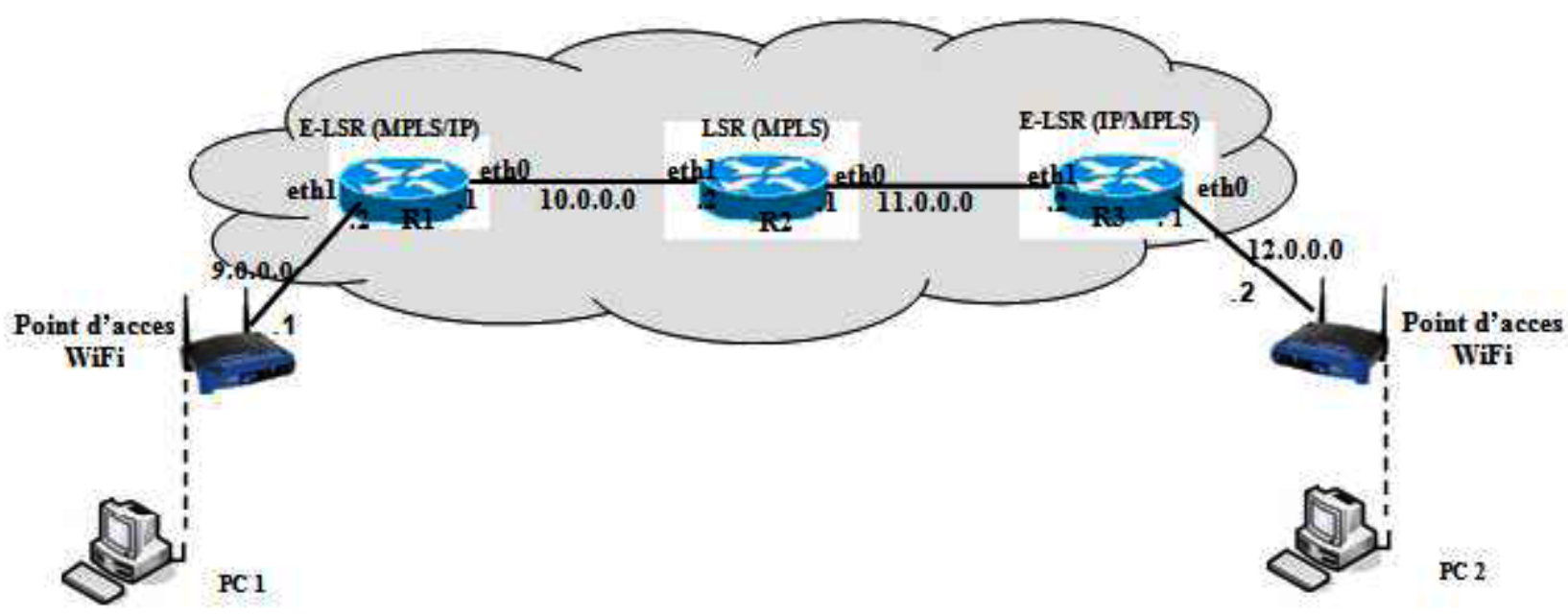

Figure 5. Mapping model

The PC1/PC2 represents the server/customer machine that will be used to transfer the FTP/UDP applications, the last component MPLS backbone constituted by the routers R1, R2 and R3.

Our focus simulations is testing some interconnection scenarios between 802.11 environments and IP QoS networks to antcipate problems found in order to implement the best scenario according to the user's needs.

The principle object is to compare the use of MPLS or/and DiffServ protocols based on some QoS criteria such as: bandwidth, delay, loss and Signaling.

We have compared performance of IP model networks and MPLS_TE. The compared parameters are Delay Variation, End to-End Delay, FTP Response Time and Packet Receive and Send. MPLS-TE results better than conventional IP network model for all the QoS parameters. The better performance of MPLS-TE is more clear, in the network congestion. All routers are usual IP routers in 
scenario 1 MPLS definition attribute is not taken into consideration and the packets are propagated by using OSPF protocol. Thus all packets are routed over the shortest path only and doesn't take into account the other two paths. In Scenario 2, MPLSTE is carrying out by generating LSPs, and defining how traffic is allocating to the corresponding LSPs. The network load is equally avoided among the three LSPs.

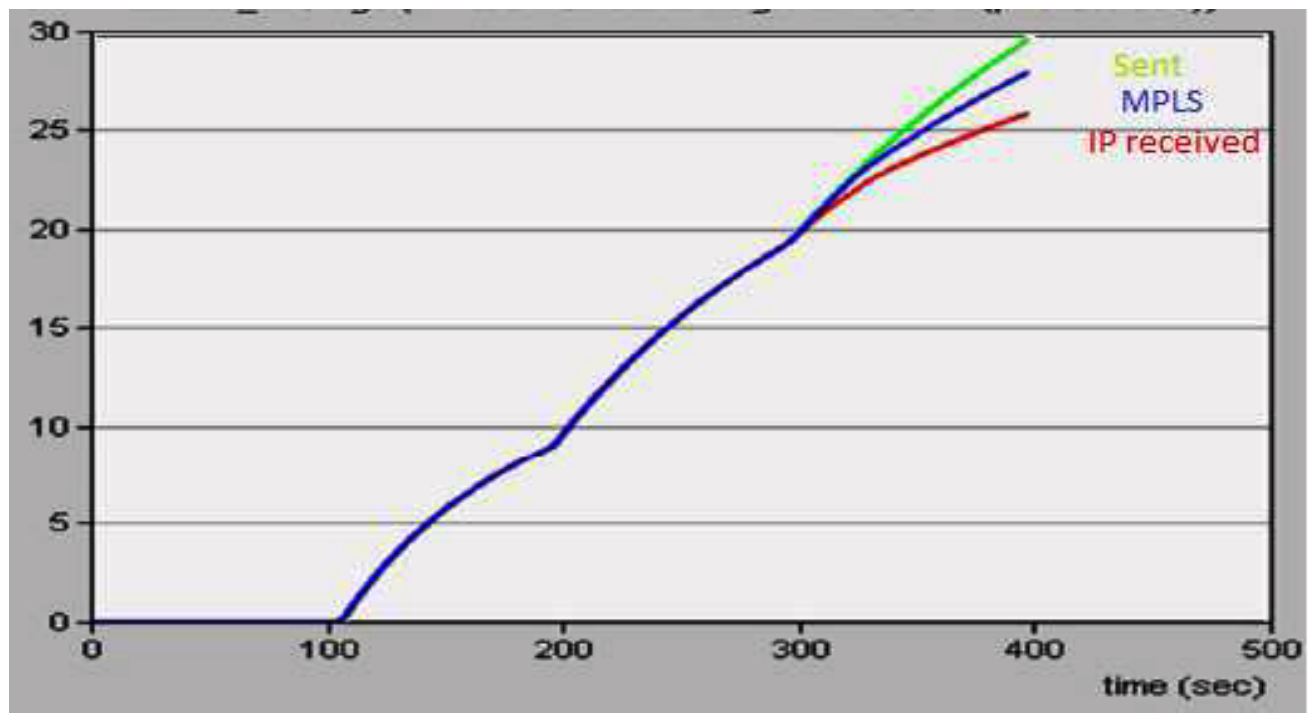

Figure 6. Video packet send and received

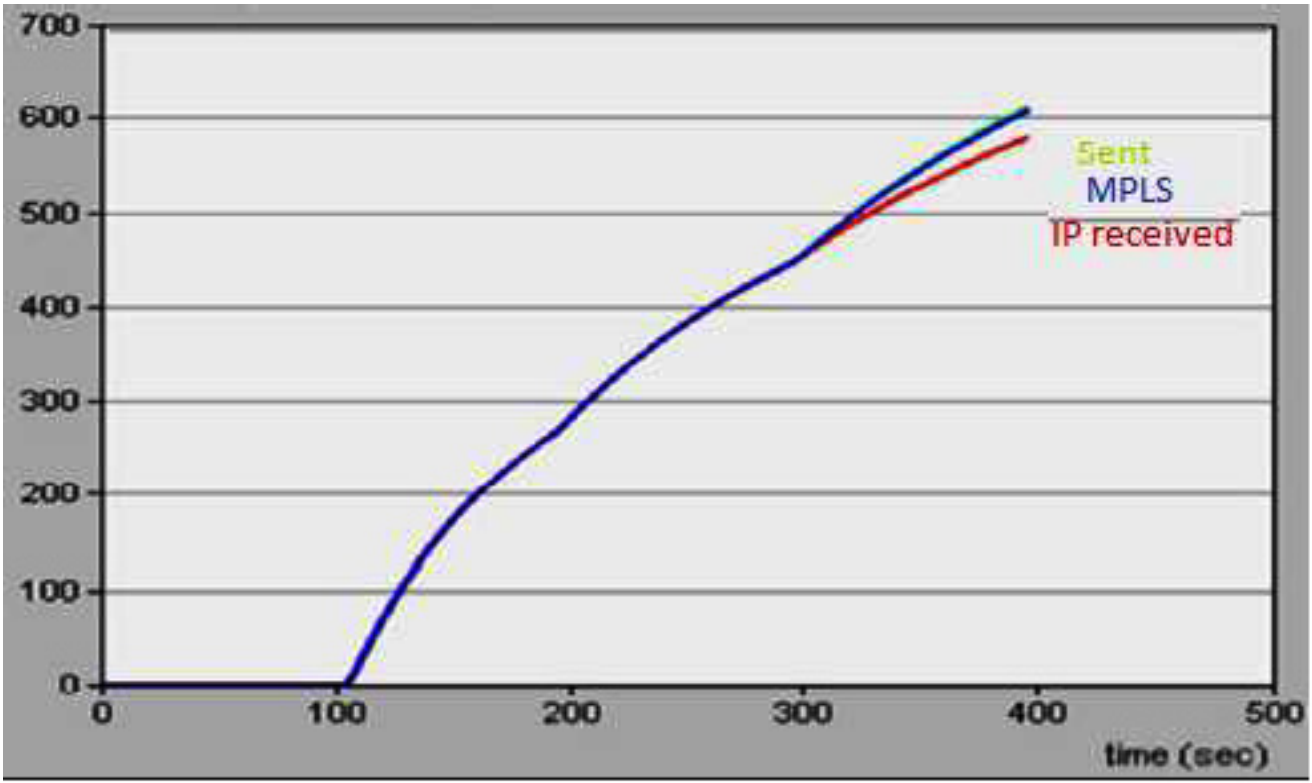

Figure 7. Voice packet send and received

Figure 7 and Figure 8 show the packets sent and received in IP networks and MPLS for both video and voice traffic. The results show that MPLS model provides more throughput compared to the IP model, and also more performance in loss packet. In the proposed system the QoS routing problem is formulated for maximizing the link stability and minimizing the cost. Fuzzy rule base is proposed to combine the various metrics such as bandwidth, End-to-End delay and number of nodes to generate a minimum cost value.

\begin{tabular}{llllll}
\hline 62 & Journal of Networking Technology & Volume & 9 & Number 2 & June 2018 \\
\hline
\end{tabular}


In load balancing, Figure 8 and Figure 9 shows the end-to-end delay of video and voice traffics. It is clear that MPLS has a minimum delay as compared to the IP model in the situation of load network. In the situation of both interactive voice the delay is less than $200 \mathrm{~ms}$ and in video the delay is less than $250 \mathrm{~ms}$. The delay or jitter variation is approximately (30-50) ms. The delay variation of video and voice traffics are shown in Figure 10 and Figure 11. The delay variation result presents that MPLS-TE has a lesser delay parameter in comparison of IP network model.

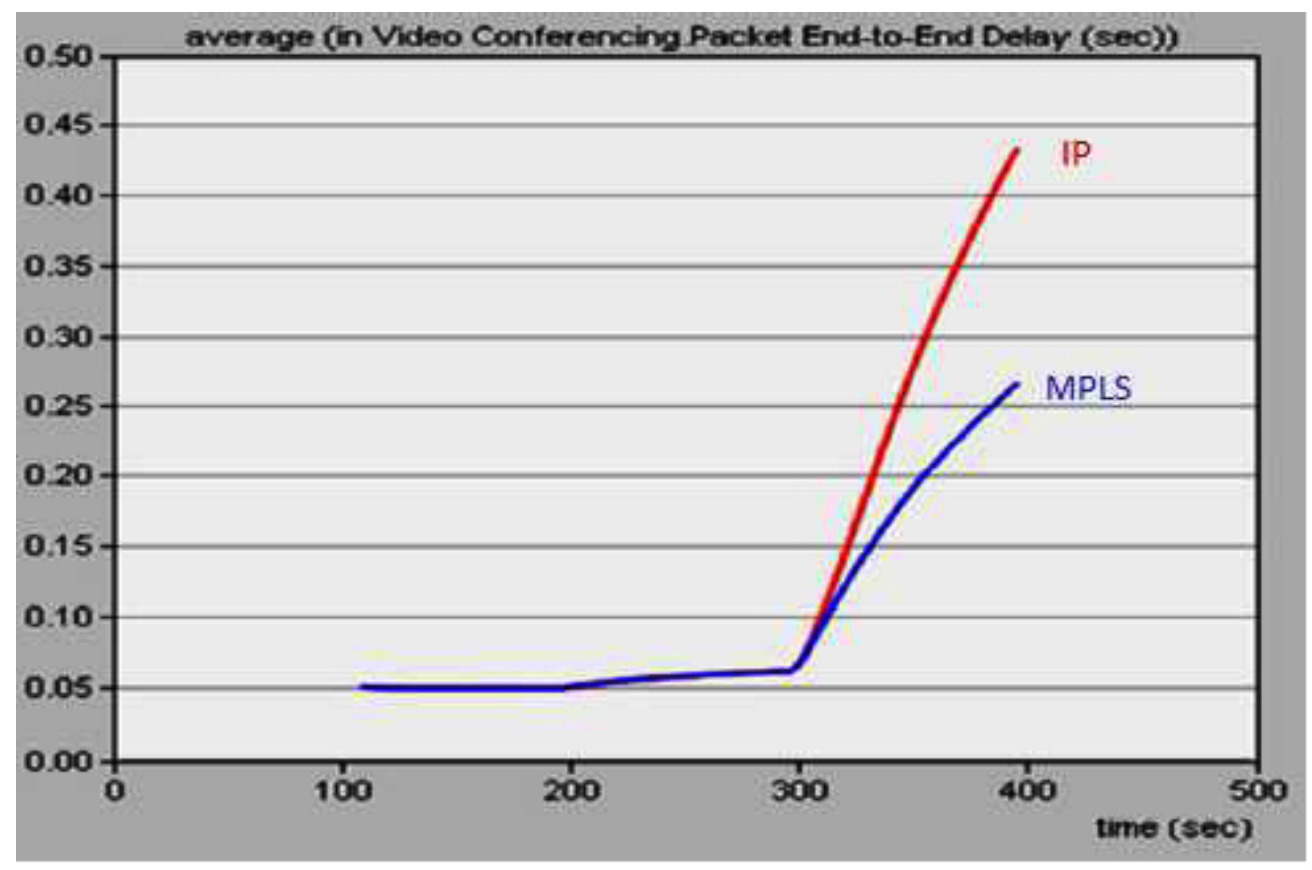

Figure 8. Video packet end to end delay

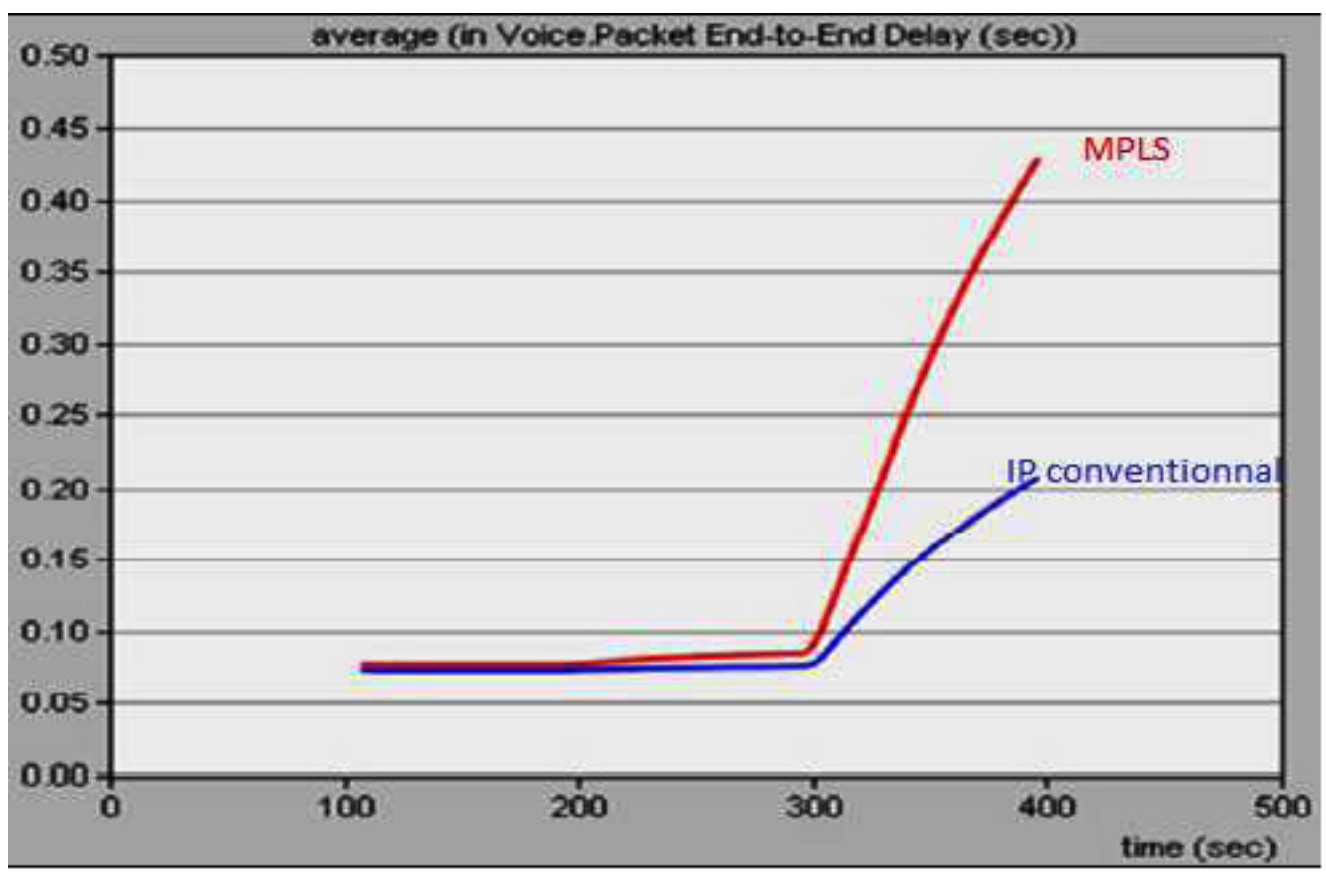

Figure 9. Voice packet end to end Delay 


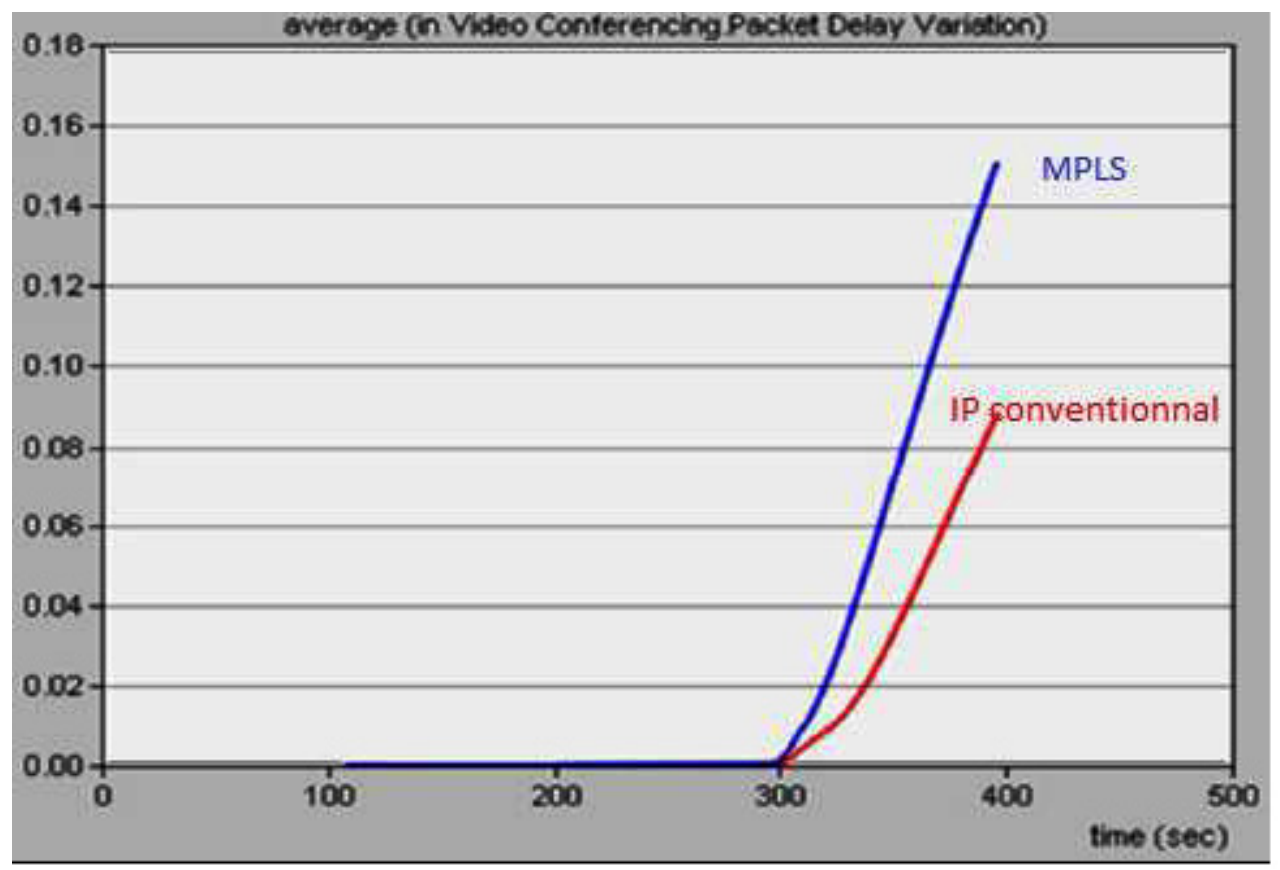

Figure 10. Video packet delay variation

\section{Conclusion}

The aim of this paper was to propose the best combination between several heterogeneous systems by used the varieties flows with a different priority, and we discussed how MPLS and 802.11e work together with Fuzzy-logic. It explained different possibilities to incorporate the IEEE 802.11e into MPLS networks, and also demonstrated the importance of our approach and its role for providing QoS specially in the interconnection networks. This work is designed to develop mechanisms capable of providing a quality of service between two mechanisms of QoS to achieving the future QoS with routers supporting many types of traffic classification and also showed the importance of our approach and its role in terms of a providing QoS the end to end. This paper designed to offer more choices of service according to Access Categories and to develop mechanisms capable for provide a quality of service between two mechanisms in order to support many types of traffic classification.

\section{References}

[1] IEEE 802.11 WG (1999). Wireless Lan Medium Access Control and Physical-Layer (PHY) Specifications 1999; standard.

[2] IEEE 802.11e (2004). Wireless Lan Medium Access Control (MAC) Enhancements for Quality of Service (QoS). 802.11e Draft $8.0,2004$.

[3] Patel, N., Patel, R. Optimizing Performance of OLSR Protocol Using Energy Based MPR Selection in MANET Sefali Prajapati; 2015 Fifth International Conference on Communication Systems and Network Technologies.

[4] Oubaha, J., Echchaachoui, A., Ouacha, A., Elkoutbi, M. (2011). New Method: Mapping of 802.11e into MPLS Domains, Conception and Experimentation, ICDIPC 2011, Part II, Communication in Computer and Information Science, 189, p. 470-483.

[5] Acharya, H. S., Dutta, S. R., Bhoi, R. (2013). The Impact of self-similarity Network traffic on quality of services (QoS) of Telecommunication Network, International Journal of IT Engineering and Applied Sciences Research (IJIEASR), 2, p. 54-60, (February).

[6] Czarkowski, Michal., Kaczmarek, Sylwester., Wolff, Maciej. (2016). Influence of Self-Similar Traffic Type on Performance of QoS Routing Algorithms, INTL Journal of Electronics and Telecommunications, 62 (1) 81-87.

[7] Rivoirard, L., Wahl, M., Sondi, P., Berbineau, M., Gruyer, D. (2017). Performance evaluation of AODV, DSR, GRP and OLSR for VANET with real-world trajectories, In: 15 th International Conference on ITS Telecommunications (ITST). 
[8] Ni, Wei., Liu, Zhenwei. (2015). A routing algorithm for Network-on-Chip with self-similar traffic, ASICON 2015 IEEE 11 th International Conference on ASIC, 3-6 (November).

[9] Oubaha, J., Habbani, A., Elkoutbi, M. (2011). New Approach: Mapping of 802.11e into MPLS Domains, The 2nd International Conference on Multimedia Computing and Systems (ICMCS'11) IEEE Xplore, p.497-502.

[10] Rivoirard, L., Wahl, M., Sondi, P., Berbineau, M., Gruyer, D. (2017). Performance evaluation of AODV, DSR, GRP and OLSR for VANET with real-world trajectories, 15th International Conference on ITS Telecommunications (ITST) 2017.

[11] Trimintzios, P. (2001). A Management and Control Architecture for Providing IP Differentiated Services in MPLS-based Networks, IEEE Communication Magazine, (May).

[12] Kirichenko, L. O., Radivilova, T. A., Kayali, E. Routing calculation value in the MPLS network based on fractal properties of traffic, Automated control systems and automation equipment.

[13] Kaur, Damanjit., Kumar, Dinesh. Comparative Analysis of MPLS Signaling Protocols, International Journal of Computer Science Trends and Technology (IJCST), 3 (4) (July - August).

[14] Ferronato, J., Antonio, M., Trentin, S. (2017). Analysis of Routing Protocols OLSR, AODV and ZRP in Real Urban Vehicular Scenario with Density Variation, IEEE Latin America Transactions, 15 (9) 1727 - 1734. 